\title{
Matéria \\ (

\section{Avaliação Radiodosimétrica através do Código MCNP-5 da Radiosteoplastia em Tumores Ósseos nos Membros}

\author{
Márcia Flávia Silveira, Tarcísio Passos Ribeiro de Campos \\ Programa de Pós-Graduação em Ciências e Técnicas Nucleares - PCTN \\ Departamento de Engenharia Nuclear, Universidade Federal de Minas Gerais - UFMG \\ Av. Antônio Carlos, 6627, Pampulha, Prédio PCA1, Belo Horizonte, MG. CEP: 31270901 \\ e-mails: marciaflavia@nuclear.ufmg.br, campos@nuclear.ufmg.br
}

\begin{abstract}
RESUMO
O presente trabalho aborda a dosimetria computacional de um caso clínico envolvendo uma criança com tumor ósseo na fíbula, onde as respectivas imagens tomográficas foram digitalizadas e tratadas no programa SISCODES, gerando assim o modelo de vóxel 3D, representativo do caso clínico, que foi transferido para o código Monte Carlo, MCNP-5. Características nucleares da fonte incorporada no compósito radioativo são apresentadas com sua respectiva atividade específica. Os resultados obtidos da distribuição espacial de dose oriunda da emissão gama do Sm-153 foi uma taxa de dose de $9,02.10^{-5}$ $\mathrm{GyhMBq}^{-1}$ e uma dose acumulada de $6,23 \cdot 10^{-3} \mathrm{GyhMBq}^{-1}$. Portanto, $7400 \mathrm{MBq}$ produzirá uma dose no tumor de $47 \mathrm{~Gy}$, compatível possivelmente com o controle local do tumor. Pode-se concluir que a adequada distribuição espacial de dose suporta o uso do protocolo denominado radiosteoplastia no tratamento de tumores ósseos de membros superiores e inferiores. A radiosteoplastia é uma técnica que está sendo avaliada no NRI - Núcleo de Radiações Ionizantes da UFMG. Trata-se de um procedimento que consiste na injeção de um biomaterial radioativo no interior da estrutura óssea afetada pelo câncer, objetivando redução da dor, aumento da resistência de carga naquela estrutura e, principalmente, o controle do tumor ou da metástase de forma local.
\end{abstract}

Palavras chave: Dosimetria computacional, radiosteoplastia, tumor ósseo, MCNP.

\section{Radiodosimetric Evaluation through MCNP-5 Code of the Radiosteoplasty on Bone Tumors in Members}

\begin{abstract}
The present paper addresses the computational dosimetry of a clinical case involving a child with tumor on the fibula, whose tomographic images were digitalized and manipulated by the SISCODES program, in order to generate a 3D voxel model, representative of the clinical case. Later, it was plugged in to the Monte Carlo MCNP-5 code and the model was used for absorbed dose prediction. Nuclear features of the cement incorporating the radionuclide composite were presented with its respectively specific activity. The spatial dose rate distribution from the gamma emission of the Sm-153 was $9.0210^{-5} \mathrm{GyhMBq}^{-1}$ and the imparted absorbed dose was $6.2310^{-3} \mathrm{GyhMBq}^{-1}$. Therefore, $7400 \mathrm{MBq}$ produced a tumor dose of $47 \mathrm{~Gy}$, suitable for the local tumor control. As conclusion, an adequate spatial dose distribution supports the application of this protocol, namely radiosteoplasty, as a therapy for bone tumors on the superior and inferior members. The radiosteoplasty is a technique that is in development in the research group NRI- Núcleo de Radiações Ionizantes at UFMG. It is a procedure that consists of the injection of a radioactive biomaterial into the bone structure affected by cancer, following pain reduction, structural resistance increasing, and mainly tumor or metastasis control in situ.
\end{abstract}

Key words: Computational dosimetry, radiosteoplasty, bone tumors, MCNP.

\section{INTRODUÇÃO}

Os tumores ósseos primários de todos os tipos são incomuns, mas, não obstante, são neoplasias importantes, porque muitas ocorrem em crianças e em pessoas jovens sendo potencialmente letais. Apesar de relativamente pouco freqüentes em relação aos de outros órgãos e tecidos (1/100.000 habitantes/ano; 1,39\% 
de todas as neoplasias), os tumores ósseos necessitam de diagnósticos e tratamentos específicos. Por outro lado, os estágios finais de muitas neoplasias malignas estão associados quase que inevitavelmente a metástases, onde o esqueleto é freqüentemente afetado. Metástases ósseas estão presentes em aproximadamente $25 \%$ de todos os óbitos decorrentes de doença maligna. Em algumas circunstâncias, o primeiro indício da presença de um tumor é uma fratura patológica súbita. Os principais tratamentos que podem ser usados em tumores ósseos são: cirurgia, quimioterapia e radioterapia $[\underline{1}, \underline{2}, \underline{3}, \underline{4}]$.

O presente trabalho introduz um novo protocolo dito radiosteoplastia para tratamento de tumores ósseos em membros. A motivação de introduzi-lo está no fato de possibilitar um tratamento localizado das lesões tumorais no osso, muitas vezes frágeis e plásticas, propiciando assim uma braquiterapia óssea. $\mathrm{O}$ desafio desta técnica é garantir que a estrutura acometida pelo tumor receba uma dose absorvida adequada para o controle tumoral, essencial para evitar a recorrência local. Agindo assim, espera-se alcançar os dois objetivos essenciais do tratamento: fazer o controle local do tumor e preservar a função motora da estrutura acometida, evitando a amputação dos membros.

Para o presente estágio de desenvolvimento da radiovertebroplastia, se faz necessário a avaliação radiodosimétrica, visto que a dose necessária para obter o controle do tumor deve ser impreterivelmente atingida. A vertebroplastia percutânea é um procedimento intervencionista que, associada à radioterapia pode ser utilizada para tratamento de neoplasias malignas da coluna [5].

O método de Monte Carlo pode ser usado para simular teoricamente um processo estatístico (como a interação de partículas nucleares com materiais) e é particularmente interessante para resolver problemas de transportes nucleares complexos que não podem ser modelados por códigos baseados em métodos determinísticos. Em transporte de partículas, a técnica de Monte Carlo consiste em seguir cada uma de muitas partículas durante toda sua trajetória até algum evento terminal como absorção, escape, entre outros. A simulação tridimensional do transporte de partículas nucleares é uma importante ferramenta para melhoria dos procedimentos radioterápicos em oncologia [ㅁ] .

O sistema SISCODES (Sistema de Códigos para Cálculos de Dose Absorvida por Método Estocástico) é uma ferramenta recentemente desenvolvida para o planejamento computacional tridimensional que funciona como interface para o código MCNP-5 (Monte Carlo N-Particle Tranport Code, versão 5). Este sistema possibilita que tratamentos radioterápicos possam ser simulados de forma tridimensional levando em consideração a heterogeneidade anatômica e morfológica das estruturas. Para a simulação do tratamento radioterápico foi construído um fantoma computacional onde o modelo de vóxel foi incorporado. Este modelo é gerado a partir da imagem digitalizada de tomografia computadorizada (TC) do paciente. O código SISCODES auxilia na preparação do modelo de vóxel e de sua conversão para o formato aceitável utilizado no código MCNP onde a simulação da interação da radiação no modelo é processada. No SISCODES a distribuição de vóxels ou a morfologia da imagem digitalizada é apresentada por diferentes tons de cinza correspondente ao tecido. O usuário identifica estes tons, informando o tipo de tecido correspondente a cada área, criando um modelo tridimensional colorido. Os tecidos disponíveis têm sua composição química e densidade mássica previamente inseridas em um banco de dados, acopladas a informações nucleares, tendo como base informações da ICRU-46 [무교 $\underline{7} \underline{8}]$.

\section{RADIOSTEOPLASTIA}

A radiosteoplastia pode ser definida como um procedimento intervencionista, minimamente invasivo, que consiste na injeção de biomaterial, dito compósito ósseo radioativo, no interior da estrutura óssea afetada pelo tumor, muitas vezes frágil e plástica, objetivando a irradiação localizada com controle local da doença, bem como a redução da dor e aumento da resistência de carga naquela estrutura. Em resumo, tal compósito é produzido pela mistura de um cimento ósseo com radioisótopo incorporado. Para os futuros estudos experimentais serão empregados o PMMA - polimetilmetacrilato como agente ligante e os macroagregados de hidroxiapatita com radionuclídeos incorporados.

O PMMA é um polímero acrílico já empregado na medicina. Como produto injetável, por exemplo, é usado na bioplastia (cirurgia plástica na estética), na forma de um gel veicular que é absorvido e eliminado. Neste caso, o colágeno auxilia na fixação do biomaterial no local implantado, impossibilitando-o de migrar. Por sua vez, a hidroxiapatita (HA) tem sido utilizada em enxertos sintéticos como substituto do enxerto ósseo em casos de lesões traumáticas, infecciosas, degenerativas, congênitas ou tumorais [ $\underline{9}, \underline{10}]$. A combinação destes dois materiais, na forma de um cimento ósseo cumpre as condições para a aplicação da radiosteoplastia.

\section{OBJETIVOS E JUSTIFICATIVA}

Este trabalho tem como objetivo estudar a viabilidade dosimétrica da radiosteoplastia fazendo uso de modelos computacionais aplicado a um caso clínico real. Estudos dosimétricos computacionais são 
desenvolvidos em um segmento do membro inferior acometido por um tumor ósseo com fins de controle desta patologia. A simulação tridimensional do transporte de partículas nucleares é empregada neste contexto.

Tal técnica em desenvolvimento pode ser uma ferramenta para controle tumoral em membros, podendo obter o controle do tumor evitando a perda do membro. A irradiação induzida pelo cimento ósseo radioativo injetado junto ao tumor e envolvendo uma margem de segurança poderá esterilizar uma porção do osso in situ acometido pelo tumor e focos próximos a lesão. A parte intacta óssea poderá então promover a revascularização da parte esterelizada, com ou sem adicional enxerto. Tal hipótese está sendo investigada experimentalmente no NRI.

\section{MATERIAIS E MÉTODOS}

Foi desenvolvido um modelo computacional tridimensional de vóxels representativo da região volumétrica em estudo. Tal modelo conhecido por fantoma computacional, reproduz através de uma matriz de vóxel parte do membro inferior específica de uma criança acometida por um osteossarcoma. O caso clínico selecionado foi o de uma criança de 15 anos, do sexo masculino, que apresentava um tumor ósseo localizado no $1 / 3$ proximal da fíbula esquerda, cujo tumor o levou ao óbito.

Este modelo foi obtido através da conversão de 12 seções tomográficas computadorizadas préselecionadas. A Figura 1 ilustra duas destas seções de TC do caso clínico escolhido evidenciando a área comprometida pelo tumor. As imagens obtidas por TC em cortes axiais foram transferidas e digitalizadas para o sistema computacional SISCODES que reproduziu uma matriz de vóxels em 32 graus de cinza. Tal matriz é a base do modelo tridimensional de vóxels da estrutura comprometida que foi estudada.
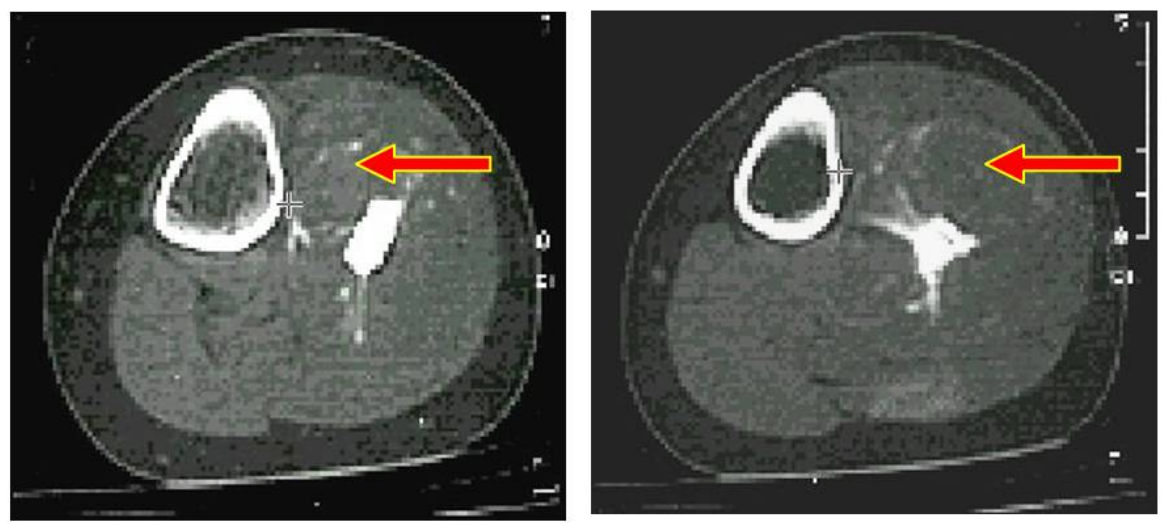

Figura 1: Ilustração de duas imagens de TC em cortes axiais de um tumor ósseo localizado na fíbula (indicado pelas setas)

A partir dos conhecimentos de anatomia dos membros inferiores, as principais estruturas anatômicas foram identificadas no modelo conforme os graus de cinza encontrados na imagem pré selecionando cada conjunto de pixels representativo de um determinado tecido. Uma cor pré-definida foi necessária para identificar cada tecido. Estas cores são usadas conforme já definidas no banco de dados de tecidos, constituinte do SISCODES. A Figura 2 mostra a interface do programa SISCODES com uma das seções anatômicas digitalizada cujo vóxels foram identificados de acordo com o banco de tecidos oferecido por este programa. Nesta figura, pode-se observar a estrutura óssea (bege), a região tumoral (lilás), o músculo (verde escuro), o tecido conjuntivo (rosa), compósito radioativo (roxo) e a pele (fina camada rosa externa). Nesta fase, a matriz de vóxel representativa da estrutura em estudo tem 152.100 elementos (130x117x10). 


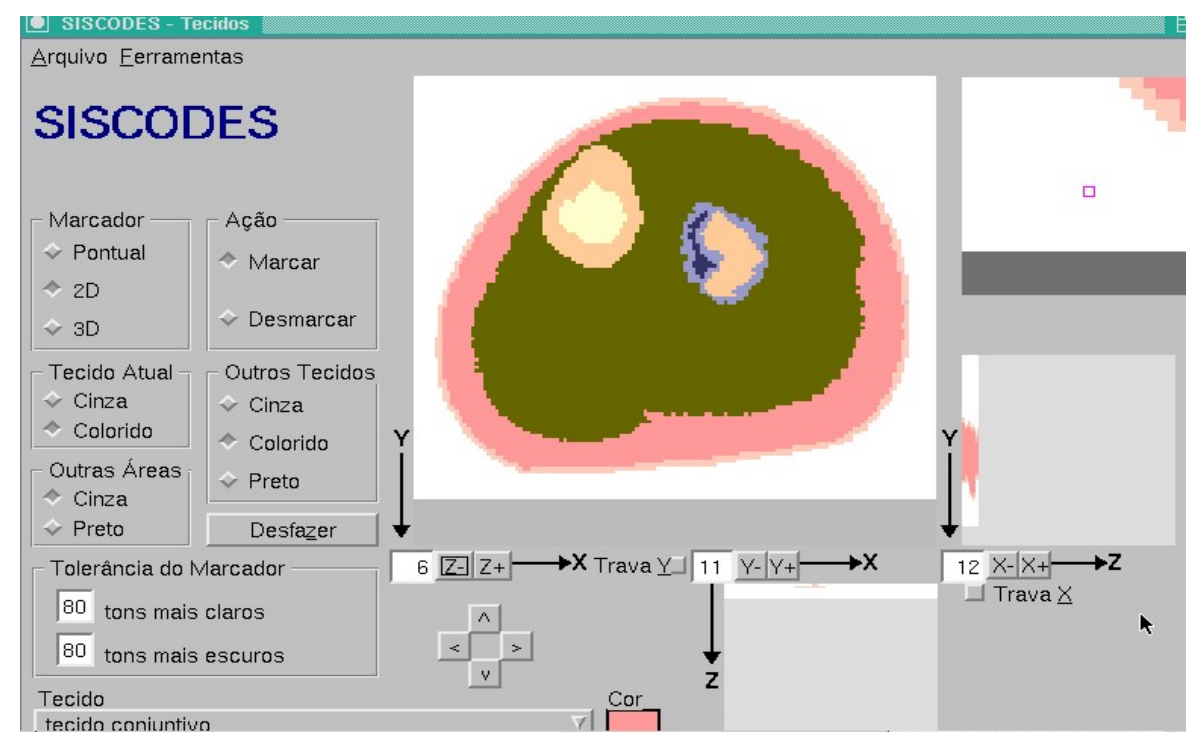

Figura 2: Ilustração do processo de criação do modelo de vóxel utilizando o programa SISCODES equivalente ao segmento da fíbula com os tecidos em cores.

Previamente foi dado a cada tecido ou estrutura uma determinada cor baseando-se nos graus de cinza evidenciados no exame tomográfico, gerando assim uma imagem de vóxel colorida. As imagens então coloridas foram tratadas pelo programa SISCODES afim de que todos os vóxels fossem identificados reproduzindo de forma análoga a anatomia e a composição elementar dos tecidos.

Este sistema, recentemente criado para calcular e estimar a dose absorvida em protocolos de radioterapia tem como objetivo estudar a dosimetria baseada na anatomia tomográfica representativa de uma região anatômica específica. A partir da criação de uma malha de vóxels, cujos tecidos são identificados em um banco de dados nucleares, o sistema exporta as informações volumétricas do modelo, produzindo os dados de entrada no código MCNP-5. A dosimetria computacional é executada através do código MCNP-5 e seus resultados são convertidos novamente para o sistema SISCODES []].

Os tecidos utilizados na etapa anterior são previamente cadastrados em um banco de dados através de uma interface WEB. A Tabela 1 apresenta a relação de tecidos com sua respectiva composição elementar e densidade mássica empregados no modelamento computacional. Relacionados aos elementos químicos, são cadastrados também os coeficientes de Kerma para fótons e nêutrons dos respectivos tecidos incorporados no banco de dados $[11, \underline{12}]$.

Tabela 1: Composição elementar dos tecidos utilizados no fantoma computacional.

Composição Elementar (\% em peso, superior a $0,1 \%)$

\begin{tabular}{l|c|c|c|c|c|c|c|c|c|c|c|c}
\multicolumn{1}{c|}{ Tecido } & $\mathrm{C}$ & $\mathrm{Ca}$ & $\mathrm{Cl}$ & $\mathrm{H}$ & $\mathrm{K}$ & $\mathrm{N}$ & $\mathrm{Na}$ & $\mathrm{O}$ & $\mathrm{P}$ & $\mathrm{S}$ & $\mathrm{Mg}$ & Dens. $\left[\mathrm{g} / \mathrm{cm}^{3}\right]$ \\
Pele & 20.4 & & 0.3 & 10.0 & 0.1 & 4.2 & 0.2 & 64.5 & 0.1 & 0.2 & & 1.09 \\
Tecido conjuntivo & 20.4 & & 0.3 & 10.0 & 0.1 & 4.2 & 0.2 & 64.5 & 0.1 & 0.2 & & 1.05 \\
Osso cortical & 15.5 & 22.5 & & 3.4 & & 4.2 & 0.1 & 43.5 & 10.3 & 0.3 & 0.2 & 1.92 \\
Osso esponjoso & 40.4 & 7.4 & 0.2 & 8.5 & 0.1 & 2.8 & 0.1 & 36.7 & 3.4 & 0.2 & 0.1 & 1.18 \\
Músculo esquelético & 14.3 & & 0.1 & 10.2 & 0.4 & 3.4 & 0.1 & 71 & 0.2 & 0.3 & & 1.05 \\
\hline
\end{tabular}

Fonte: http://nri.nuclear.ufmg.br [11].

Observa-se que as ligações químicas, por exemplo, das proteínas, não interferem no transporte de partículas nucleares dentro do espectro energético da fonte em questão. Nesta tabela são considerados apenas os elementos que têm concentração em peso superior a $0,1 \%$ visto que, elementos com valores inferiores 
pouco influenciará no transporte de partículas nucleares na região. A composição do ar externo foi tomada sendo constituída de nitrogênio $(75,5 \%)$ e oxigênio $(23,2 \%)$, com densidade mássica de 0,0012 g.cm ${ }^{-3}$.

$\mathrm{Na}$ simulação foi empregado o radionuclídeo Sm-153 que foi distribuído na região definida para o compósito ósseo incorporado. Assim, a fonte está distribuída em um conjunto de vóxels arbitrariamente definidos junto à região tumoral. O Samário-153 é produzido desde 1993 pelo Instituto de Pesquisas Energéticas e Nucleares (IPEN). Este radionuclídeo tem meia-vida de 46,3 hs, emissão de partículas $\beta^{-}$(beta) de energias máximas de $808 \mathrm{keV}(17,5 \%), 705 \mathrm{keV}(49,6 \%)$ e $635 \mathrm{keV}(32,2 \%)$, entre outras, além de raios $\gamma$ (gama) e raios-X. Entre as gamas emitidas estão 103,18 keV (30\%), 69,67 (4,85\%) e raios-X de 6,4 keV (2.85\%), 40,9 keV (17,9\%), 41,5 keV (32,2\%), 46,9 keV (3,17\%), 47,0 (6,13\%), 48,2 (2,03\%). Trata-se de um radiofármaco que permite condições ideais para produção de imagens por cintilografia quando aplicado de forma sistêmica. O Sm-153 é de fácil ativação neutrönica em reatores de pesquisa, devido as elevadas seções de choque térmica e epitérmica, 206 e 2970 barns, respectivamente [14]. Esta escolha é atrativa considerando a emissão de raios gamas e X de baixa energia, sua baixa meia vida, e emissão de raios betas que permanecem no leito do tumor. Ambas as emissões betas, raios-gama e X contribuem com a dose no tumor $[\underline{13}, \underline{14}, \underline{15}]$. A meia-vida que caracteriza o decaimento radioativo do nuclídeo samário-153 é de 46,3 horas; entretanto sua eliminação corpórea produz uma meia-vida biológica de 50,6 dias [16].

No presente estudo de distribuição espacial de dose serão consideradas apenas as emissões de raios $\gamma$ do Sm-153. As emissões de raios $\beta$ estão limitadas a um range de 2 a $3 \mathrm{~mm}$ afastados dos vóxels identificados como compósito ósseo radioativo. Assim, estudos de distribuição espacial de dose devido às emissões beta terão baixa resolução espacial em uma malha discretizada na ordem de milímetros. Entretanto, se torna evidente dizer que todas as betas serão emitidas e absorvidas junto ao tumor.

\section{RESULTADOS}

O fantoma computacional de vóxel incorporado no código MCNP-5, juntamente com os dados da simulação são os resultados do presente estudo. O cálculo da taxa de dose foi processado em todo o modelo de vóxel, entretanto foi impressa em uma única seção representativa. A Figura 3 (à esquerda) ilustra a imagem em corte do fantoma computacional. Observa-se, em azul claro, a região onde se recebeu o cimento ósseo radioativo; em roxo, a região do tumor; em rosa, a parte do osso não acometido pelo tumor; em laranja, a tíbia; em amarelo, a musculatura; em verde, o tecido epidérmico e em azul escuro, a pele. Observa-se que o padrão de cor no SISCODES é diferente do adotado na parte gráfica do MCNP-5. No caso em estudo foi considerada uma malha representada por 130x117x10 vóxels, com discretização volumétrica de $13 \mathrm{~cm}, 12 \mathrm{~cm}$ e $5 \mathrm{~cm}$. O código MCNP-5 forneceu para cada vóxel da malha a taxa de dose absorvida em função da atividade injetada, em unidades de $\mathrm{Gyh}^{-1} \mathrm{MBq}^{-1}$. Ainda na Figura 3 (à direita) pode-se observar a imagem do fantoma analítico destacando a região que está sendo tratada em forma de vóxel pelo programa computacional.
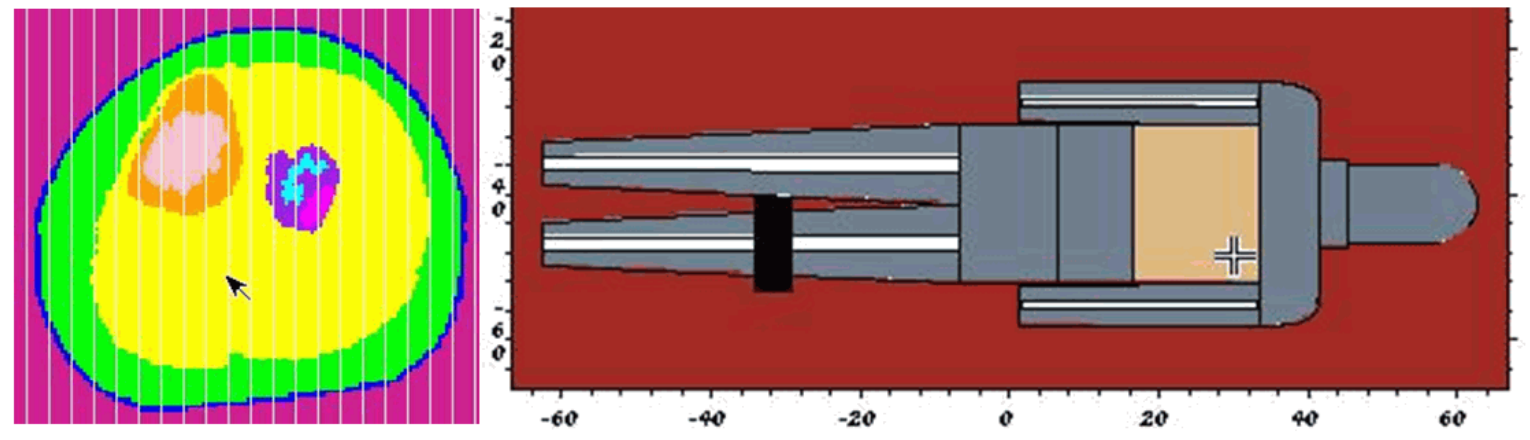

Figura 3: Observa-se uma seção em corte do modelo tridimensional de vóxel (à esquerda), mostrando a equivalência anatômica; e à direita o corte longitudinal do fantoma analítico com vista da região onde está sendo incorporado o fantoma de vóxel.

A Figura 4 ilustra uma seção transversal da região tomada pelo tumor, entre as 15 seções tomográficas axiais estudadas. As cores ilustram as regiões: 1-5\% (azul), 5-10\% (verde), 10-50\% (amarelo), $50-80 \%$ (laranja) e $80-100 \%$ (vermelho) da máxima dose absorvida. Esta figura é obtida na interface gráfica de saída do sistema SISCODES. Nesta saída, o modelo tomográfico é projetado em azul escuro, de forma transparente, para não interferir com a distribuição de taxa da dose colorida. 


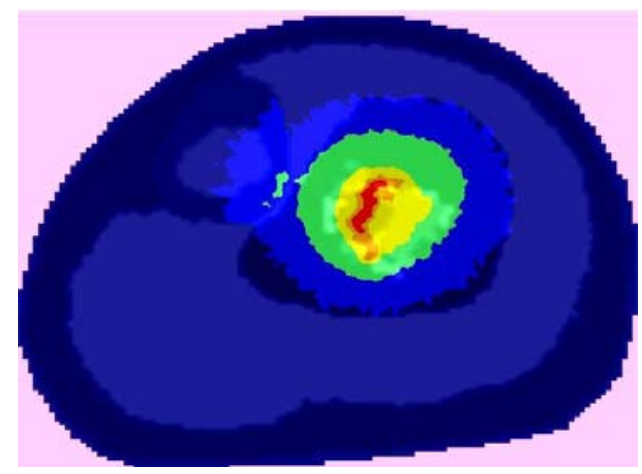

Figura 4: Imagem da distribuição espacial da dose na seção transversal média da região tomada pelo tumor ósseo.

A taxa de dose máxima atingida no plano estudado foi de $9,02.10^{-5} \mathrm{Gyh}^{-1} \mathrm{MBq}^{-1}$ e a dose mínima foi de $1,0.10^{-7} \mathrm{Gyh}^{-1} \mathrm{MBq}^{-1}$ na seção estudada. Tomando estes valores, estima-se que a dose absorvida acumulada total em Gy.MBq ${ }^{-1}$ é obtida através da expressão: $1,44 \mathrm{~T}_{1 / 2}(\mathrm{hr})$ vezes a taxa de dose $\left(\mathrm{Gy}^{-1} \mathrm{~h}^{-1} \mathrm{MBq}^{-1}\right)$, onde $\mathrm{T}_{1 / 2}$ representa a meia vida em horas do radionuclídeo. Assim, a dose acumulada no implante será de $6,23.10^{-3}$ Gy. $\mathrm{MBq}^{-1}$. Para uma atividade de $7400 \mathrm{MBq}(200 \mathrm{mCi})$, a dose atingida no implante será de 46,6Gy, equivalente a emissão gama do Sm-153. Para uma atividade de $2 \mathrm{Ci}(370 \mathrm{GBq})$, a dose máxima será de $466 \mathrm{~Gy}$, sendo que a região de $10-5 \%$ desta dose (região verde) receberá 46,6 Gy, e de 5-1\% receberá 23,3 Gy. Valores equivalentes são adequados para o controle local do tumor obedecendo a uma margem de segurança. A emissão beta do Sm-153 será totalmente absorvida no implante e não foi avaliada no momento. Entretanto espera-se que no tumor a dose devido à emissão beta seja no mínimo 10 vezes superior à dose da componente gama.

\section{CONCLUSÕES}

No presente estudo estimou-se que $0,2 \mathrm{Ci}$ é uma atividade adequada para atingir controle no tumor ósseo do paciente em estudo, e $2 \mathrm{Ci}$ atingirá doses absorvidas de 23,3 Gy dentro de uma margem de raio de $2 \mathrm{~cm}$, tomando o cimento ósseo como referência. Pelo presente estudo computacional foi possível definir a atividade do radionuclídeo a ser utilizado na técnica de implante.

Em termos comparativos, a técnica de redução de dor óssea utilizando um radiofármaco sistêmico dito EDTMP-153 emprega uma dose prescrita de 3,0 $\mathrm{mCi} / \mathrm{Kg}$, que representa 0,25 $\mathrm{Ci}$ para um paciente de $70 \mathrm{~kg}$. Esta atividade representa $1 / 4$ da atividade preconizada, entretanto, no implante do cimento ósseo o radionuclídeo é implantado localmente, enquanto que no emprego do EDTMP-153 a aplicação é sistêmica. Desta forma, os efeitos deletérios das duas técnicas serão distintos.

Para conhecimento, o EDTMP-153 é aplicado por infusão "em bolus" via endovenosa, em doses variáveis de 0,5 a $3,0 \mathrm{mCi} / \mathrm{Kg}$ peso, e logo após sua administração aproximadamente $50 \%$ da atividade empregada se fixa em toda a estrutura óssea, havendo maior afinidade nas regiões metastáticas do que no tecido ósseo normal, em média de 4,8:1, com variações de 4:1 a 17:1. O restante da dose empregada, quase $50 \%$, apresenta eliminação renal, no período variável de 6 a 8 horas. É interessante ressaltar que a fixação da atividade utilizada no tecido mole não excede 1 a $2 \%$ da dose total empregada. A excreção do Samário-153EDTMP ocorre preferencialmente por via urinária, e uma atividade emitida que não ultrapassa os limites de radiorresistência dos rins e bexiga $[\underline{16}, \underline{17}]$.

Em contraste, a radiosteoplastia emprega o Sm-153 incorporado no cimento ósseo permitindo que se fixe no local do tumor. Através do implante local do cimento radioativo solidificado, os produtos do decaimento do Sm-153 ficam confinados na região de interesse não se espalhando pelo corpo humano. $\mathrm{O}$ radionuclídeo não está livre para circular no sistema vascular. Os efeitos deletérios para o paciente quando o radionuclídeo é aplicado a nível sistêmico não ocorrerão neste caso. Outros efeitos deletérios locais devido à irradiação dos tecidos sadios não acometidos pelo tumor são possíveis de ocorrer, entretanto, a indicação de tais possíveis efeitos deletérios só será concretizado após estudos in vivo.

\section{AGRADECIMENTOS}

Os autores agradecem especialmente a CAPES pela concessão de bolsa para a realização do curso de mestrado, colaborando desta forma para a concretização deste trabalho. 


\section{BIBLIOGRAFIA}

[1] RUBIN, E., FARBER, J.L., Patologia, ed. 3, Guanabara Koogan, Rio de Janeiro, 2002.

[2] BRASILEIRO FILHO, G., Bogliolo Patologia, ed.6, Guanabara Koogan, Rio de Janeiro, 2000.

[3] SUTTON, D., Tratado de Radiologia e Diagnóstico por Imagem, ed. 6, Revinter, Rio de Janeiro, 2003.

[4] COTRAN, R.S., KUMAR, V., COLlinS, T., Robbins - Patologia Estrutural e Funcional, ed., 6, Guanabara Koogan, Rio de Janeiro, 2000.

[5] MACEDO, R.A., CAMPOS, T.P.R., "Radiovertebroplastia para Tratamento de Metástases Ósseas da Coluna Vertebral”, In: International Nuclear Atlantic Conference - INAC 2005, pp.1-5, Santos, São Paulo, 2005.

[6] MCNP, MCNP - A General Monte Carlo N-Particle Transport Code, Version 5, X-5 Monte Carlo Team, Los Alamos National Laboratory, 2003.

[7] TRINDADE, B.M., "Desenvolvimento de Sistema Computacional para Dosimetria em Radioterapia por Nêutrons e Fótons Baseado em Método Estocástico - Siscodes”, Tese M.Sc., Universidade Federal de Minas Gerais, Belo Horizonte, MG, Brasil, 2004.

[8] ICRU-46, "Photon, Electron, Proton and Neutron Interaction Data for Body Tissues", International Commission on Radiation Units and Measurements, Report 46, Bethesda, 1992.

[9] CBM - CENTRO MUNDIAL DE BIOPLASTIA, http://www.clinicanacul.com.br, acessado em março de 2006.

[10] DELFINO, H.L.A., CONTI, O., VELLUDO, M.A.S.L., "Estudo Experimental da Integração Óssea da Hidroxiapatita", Revista Brasileira de Ortopedia, USP, v. 33, n. 9, pp. 713-718, 1998.

[11] SISCODES - SISCODES ADMINISTRATIVO, http://nri.nuclear.ufmg.br, acessado em maio de 2006.

[12] TRINDADE, B.M., CAMPOS, T.P.R., “Imagem Transformation from CT or MRI to a Tissue's Voxel Model Appied to Oncology”, In: IX Encontro de Modelagem Computacional, Belo Horizonte, Minas Gerais, pp. 1-8, 2006.

[13] UNGRIN, J., JOHNS, M.W., “The decay of Sm-15”, Nuclear Physics, pp. 127-353, 1969.

[14] SCHOTZIG, U., SCHONFELD, E., GUNTHER, E., KLEIN, R., SCHNADER, H., “Standardization and Decay Data of Sm-153 Applied Radiation and Isotopes”, v. 19, n. 51, pp. 169-175, 1999.

[15] CLUNIE, G., LUI D., "Clinical Outcome after One Year Following Samarium-153 Particulate Hydroxyapatite Radiation Synovectomy”, Scandinavian Journal of Rheumatology, v. 25, n. 6, pp. 360-6, 1996.

[16] ZEQUI, S.C., FONSECA, F.P., BACHEGA JR, W., et al., "Emprego do Samário-153-EDTMP no Tratamento do Adenocarcinoma de Próstata Avançado, Resultados Preliminares”, Brazilian Journal of Urology, v. 25, pp. 214-220, 1999.

[17] EARY, J.F., COLLINS C., STABIN, M., et al., "Samarium-153-EDTMP Biodistribution and Dosimetry Estimation”, Journal of Nuclear Medicine, v.34, n.7, pp1031-6, 1993. 